\title{
RELACIONES ENTRE DECODIFICACIÓN, CONOCIMIENTO LÉXICO-SEMÁNTICO E INFERENCIAS EN NIÑOS DE ESCOLARIDAD PRIMARIA
}

\author{
RELATIONS BETWEEN DECODING, LEXICAL-SEMANTIC KNOWLEDGE AND INFERENCES IN \\ PRIMARY SCHOOL CHILDREN
}

\author{
María Micaela Villalonga Penna* , Constanza Padilla Sabaté** y Débora Burin ${ }^{* * *}$ \\ *Magister en Psicología Cognitiva y Aprendizaje. Becaria del Consejo Nacional de Investigaciones Científicas \\ y Técnicas (CONICET). E-Mail: micavillalonga@yahoo.com.ar \\ ${ }^{* *}$ Profesora y Doctora en Letras. Profesora Asociada en la Facultad de Filosofía y Letras de la Universidad de \\ Tucumán (UNT) y Miembro de la Carrera del Investigador Científico del Consejo Nacional de Investigaciones \\ Científicas y Técnicas (CONICET). \\ ***Doctora en Psicología. Profesora Adjunta de la Facultad de Psicología de la Universidad de Buenos Aires (UBA) \\ y Miembro de la Carrera del Investigador Científico del Consejo Nacional de Investigaciones \\ Científicas y Técnicas (CONICET). \\ Instituto de Investigaciones del Lenguaje y la Cultura (INVELEC - CONICET), Facultad de Filosofía y Letras de la \\ Universidad Nacional de Tucumán (UNT). \\ Corrientes 318, $5^{\circ}$ Piso, Dpto. A (4000) San Miguel de Tucumán - Tucumán, República Argentina.
}

\section{RESUMEN}

La comprensión lectora es un complejo proceso que se asienta en tres habilidades básicas interrelacionadas: la decodificación, el conocimiento léxico-semántico y los procesos de integración textual. Investigaciones previas han indicado que las dificultades en la comprensión lectora pueden deberse a la decodificación ineficaz, las carencias en el conocimiento léxico-semántico y los problemas para realizar inferencias. Los objetivos del trabajo que se informa fueron estudiar las relaciones entre las habilidades de la comprensión lectora y estudiar la contribución de las habilidades de decodificación y del conocimiento léxicosemántico, a la realización de inferencias textuales. Participaron 94 niños de escolaridad primaria de 8 a 10 años de edad. Se administraron las subpruebas de Identificación de Letras y de Procesos Léxicos de la Batería de Evaluación de los Procesos Lectores (Cuetos, Rodríguez, Ruano \& Arribas, 2010), la adaptación del Subtest de Vocabu- lario de la Escala de Inteligencia de Wechsler para Niños de Contini, Figueroa, Cohen Imach y Coronel de Pace (2000) y las Tareas de Inferencias de Cain y Oakhill (Canet Juric \& Burin, 2006). Los resultados indicaron correlaciones moderadas a altas y significativas entre las habilidades lectoras. Las habilidades de decodificación y conocimiento léxico-semántico explicaron el $51 \%$ de las inferencias, pero la única que realizó una contribución significativa fue el conocimiento léxico. En el caso de las inferencias puente, el conjunto de las variables explicó el $48 \%$ de éstas y en el de las elaborativas, el 45\%. En ambos casos, el único predictor que hizo una contribución significativa fue el conocimiento léxico. En conclusión, las habilidades lectoras se hallan relacionadas y el vocabulario es un componente básico para las habilidades de mayor nivel como las inferencias.

Palabras clave: Decodificación; Vocabulario; Inferencias; Comprensión; Escolaridad primaria. 
ABSTRACT

Reading comprehension is a complex process which lies upon three interrelated basic skills: decoding, lexical semantic knowledge and wordto-text integration processes. Decoding entails the reader's ability to recognize grapheme-phoneme correspondence. Lexical semantic knowledge refers to the knowledge of words in a given language, and it includes both word identification, i.e., visual processing of the graphemes' shapes and lexical activation; and semantic awareness, i.e., knowledge of meaning.

Decoding and lexical semantic knowledge are deemed as pertaining to a lower level in relation to integration, in which category we include such skills as inference - generation. Previous research, notably in England, the United States of America and Argentina, has shown that difficulties in reading comprehension may be due to faulty decoding, poor lexical semantic knowledge and inferencemaking failures. Although each of these skills is necessary to achieve comprehension, none of them alone can guarantee it. It is considered that decoding and lexical knowledge are associated skills, and influence each other. The effects of decoding in text comprehension are mediated by knowledge about the meaning of words and the effects of comprehension in decoding depend on the achievement of sufficient word meanings.

Thus, the aims of this paper were to study the relations among reading comprehension skills and to study how decoding skills and lexical semantic knowledge contribute to inference-making generation. In broad terms, the hypotheses were reading comprehension skills are interrelated and decoding skills and lexical knowledge are significant predictors of inference-making generation.

This survey was conducted among 94 primary school children of an average age of 8 to 10 years old. They were given the Letter Recognition and Lexical Processes Tests from the PROLEC Test Battery, the Vocabulary Subtest from Wechsler Intelligence Scale for Children (WISC-III) and the Inference-making Ability Assessments from Cain and Oakhill (Canet Juric \& Burin, 2006).

Results have shown that in the decoding assessment, the highest number of errors and correct answers are found in pseudo word reading. Regarding lexical knowledge, the highest average of right answers is found in word reading. As regards wordto-text integration, the average number of inferences incorrectly drawn is 14.62 . Since the tests we used measured right and wrong answers, indices of decoding and lexical knowledge were created. Moreover, our findings suggest significant and moderate-to-strong relations among reading comprehension skills.

Decoding skills and lexical semantic knowledge have accounted for $51 \%$ of inferences. However, lexical knowledge alone has made the most significant contribution. The set of variables has accounted for $48 \%$ of coherence inferences and $45 \%$ of elaborative inferences. The only significant predictor of both types of inference-making was lexical knowledge.

To conclude, as posited by international research and our first hypothesis, comprehension skills are interrelated. Taking into consideration our second hypothesis, our findings suggest that comprehension skills account for inference-making generation and that lexical knowledge reveals itself as a predictor of the children's potential ability for inference-making. In other words, vocabulary is a basic component of more complex skills such as inference. This is consistent with what international research suggests.

As regards the performance of the general sample in the assessed comprehension skills, it resembles that of the child population of the same age, surveyed in Tucumán and in. Taking into consideration the way in which comprehension skills relate to and affect each other could allow us to develop intervention programs which would help improve the performance of children who demonstrate text comprehension difficulties.

Key words: Decoding; Vocabulary; Inferences; Comprehension; Primary school.

\section{INTRODUCCIÓN}

La consecución de las trayectorias escolares de los niños depende en gran parte, de la adquisición de habilidades lectoras. Diversas investigaciones del ámbito angloparlante y nacional han señalado las dificultades que 
presentan los niños de escolaridad básica o primaria en relación a las habilidades lectoras y en consecuencia, para comprender lo que leen. Evaluaciones realizadas en países de Europa, Latinoamérica, Asia, Canadá y Estados Unidos (PISA, 2009, 2012), y por el Ministerio Nacional de Educación y el Ministerio de Educación de la provincia de Tucumán, han detectado importantes dificultades en la comprensión lectora (DINIECE, 2007; Serrano \& Canigia, 2009). La comprensión de un texto implica la construcción de una representación mental integrada y coherente de la información que el texto vehiculiza (Abusamra, Ferreres \& Raiter, 2010; Belinchón, Igoa \& Riviére, 1992; Gernsbacher, Varner \& Faust, 1990; Gutierrez, Elosúa, García Madruga, Gárate \& Luque, 1999; Kintsch, 1998; León, 2004; Molinari, 2008; Nation, 2005). Diversos autores han enfatizado la relevancia de las habilidades implicadas en el desarrollo de la comprensión lectora (Marder, 2011; McCandliss, Beck, Sandak \& Perfetti, 2003; Nation, 2005; Perfetti, 2010a; Perfetti \& Hogaboam, 1975). A la hora de explicitar los factores que intervienen en la comprensión lectora, proponen una serie de aptitudes interrelacionadas que intervienen sobre la información que provee el texto y la que aporta el lector, viabilizando la construcción del sentido de un texto (Nation, 2005; Perfetti, 2010a; Perfetti, Landi \& Oakhill, 2005). Nation (2005) y Perfetti, Landi y Oakhill (2005) destacan las habilidades de decodificación, conocimiento léxico-semántico o vocabulario y los procesos de integración textual. En el marco de estos desarrollos, el aprendizaje de la lectura ha sido conceptualizado como un proceso altamente interactivo que, en el caso de los niños, hace necesario el desarrollo de las habilidades interconectadas antes mencionadas (Hulme, Snowling, Caravolas \& Carroll, 2004; Nation, 2005; Perfetti, 2010a).

La decodificación consiste en la habilidad del lector para realizar la correspondencia de los grafemas con los fonemas, es decir, establecer las regularidades en la relación entre las palabras del lenguaje oral y su representación gráfica alfabética (McCandliss et al., 2003; Nation, 2005; Parodi, Peronard \& Ibañez, 2010; Perfetti, 2010b; Perfetti \& Hogaboam, 1975; Perfetti, Van Dyke \& Hart, 2001; Shankweiler et al., 1999). Investigaciones del ámbito angloparlante dieron a conocer la importancia de la automatización del proceso de decodificación en la comprensión textual en el caso de niños de escolaridad básica (McCandliss et al., 2003; Perfetti \& Hogaboam, 1975) e indicaron que cuando la decodificación es lenta, ineficiente e implica un gran esfuerzo, el lector dirige sus recursos cognitivos a la identificación de letras y a la relación con el sonido correspondiente, dejando de lado la construcción del significado de lo que lee (Cain \& Oakhill, 2003; McCandliss et al., 2003; Nation, 2005; Perfetti \& Roth, 1980; Shankweiler et al., 1999).

Además, estudios internacionales (Hulme et al., 2004; Liberman, I.Y., Shankweiler \& Liberman, A.M., 1989; McCandliss et al., 2003; Nation \& Snowling, 2004; Shankweiler et al., 1999) y nacionales (Borzone de Manrique \& Signorini, 1994; Diuk \& Ferroni, 2009; Fumagalli, Wilson \& Jaichenco, 2010; Maldez, 2010) propusieron que la decodificación y la adquisición de la lectura en sus diferentes etapas, se asientan en el desarrollo de las habilidades fonológicas ya que éstas facilitan la identificación de los grafemas. Las investigaciones locales evidenciaron que en educación primaria o básica, la sensibilidad fonológica, la memoria fonológica, el conocimiento de correspondencias entre grafemas y fonemas, la denominación rápida de vocales y el vocabulario, pueden considerarse como factores que en parte determinan la posibilidad de comprender lo leído, como predictores del desempeño lector de los niños y como diferenciadores entre buenos y malos lectores (Andrés, Canet Juric \& Introzzi, 2009; Borzone de Manrique \& Signorini, 1994; Diuk \& Ferroni, 2009; Diuk, Signorini \& Borzone, 2003; Fumagalli et al.,, 2010; Maldez, 2010; Plana \& Fumagalli, 2013; Urquijo, Canet Juric, Andrés \& Ané, 2006). Además, la relación entre la decodificación y la comprensión lectora podría estar mediada por el conocimiento léxico y por la memoria de trabajo verbal (Cain, Oakhill \& Bryant, 2000, 2003). 
El conocimiento léxico-semántico o vocabulario se refiere al conocimiento de palabras propias de un idioma y abarca una serie de aspectos o subhabilidades del procesamiento léxico, por lo menos el reconocimiento de palabras y las habilidades semánticas (Nation, 2005). El reconocimiento de palabras implica el procesamiento visual de las formas de los grafemas que conducen a la activación léxica (Perfetti, 2010b; Perfetti, van Dyke \& Hart, 2001). Las habilidades semánticas hacen referencia al conocimiento acerca del significado de las palabras, lo cual parece constituir un factor necesario para lograr la comprensión textual (Nation, 2005; Perfetti, 2010a). Estudios internacionales mostraron que los sujetos que poseen un conocimiento léxico sistematizado, interconectado y organizado, logran comprender mejor lo leído, que aquellos que no lo poseen (Nation, 2005; Perfetti, 2010b; Perfetti et al., 2001). En niños de escolaridad básica o primaria, encontraron que a nivel léxico, los lectores hábiles activan con mayor precisión y rapidez la información ortográfica y fonológica que los lectores menos hábiles (Booth, MacWhinney \& Perfetti, 1999). Además, tanto los estudios internacionales (Nation \& Snowling, 1998, 2004; Oakhill, Cain \& Bryant, 2003; Perfetti, 2010a) como los realizados en Argentina (Andrés et al., 2009; Ferreres, Abusamra, Casajús \& China, 2011; Ferreres, Abusamra \& Squillace, 2010; Urquijo, 2009; Urquijo et al., 2006) mostraron que los lectores menos hábiles tenían un bajo rendimiento en la lectura de palabras frecuentes y poco frecuentes y en el establecimiento de relaciones de significado como sinonimia, paronimia y antinomia, lo cual daría cuenta de dificultades en los procesos semánticos.

La decodificación y el conocimiento léxico-semántico se suelen considerar habilidades de orden inferior en relación al nivel de integración del texto en el que se identifican habilidades como la realización de inferencias, el monitoreo de la comprensión y la sensibilidad a la estructura de la historia (Cain \& Oakhill, 2003; Cain, Oakhill, Barnes \& Bryant, 2001; Canet Juric, 2009; Nation, 2005; Perfetti, et al., 2005). Las inferencias permiten al lector identificar las relaciones entre las partes del texto y relacionar el texto con sus conocimientos previos, es decir que se basan tanto en el conocimiento del lenguaje como en el conocimiento del mundo (Kintsch, 1998; León, 2003; Molinari, 2008). Las investigaciones sobre las habilidades inferenciales en niños demostraron que alrededor de los 7 u 8 años, éstas se presentan como uno de los factores que determinan la comprensión textual (Canet Juric, 2009; León, 2003; Nation, 2005; Oakhill, 1982, 1983). En este sentido, estudios internacionales mostraron que los malos lectores de edad infantil no infieren espontáneamente, presentan dificultades para realizar las inferencias necesarias para construir una representación integrada y coherente del texto y tienen un desempeño más bajo en la realización de las inferencias cohesivas y elaborativas (Cain \& Oakhill, 1999; Cain et al., 2001; Oakhill, 1982, 1983). En consonancia con los estudios citados, en las investigaciones argentinas los malos lectores tendían a presentar dificultades para realizar inferencias puente y elaborativas (Andrés et al., 2009; Borzone, 2005; Borzone \& Silva, 2007; Canet Juric, Andrés, Urquijo \& Ané, 2007; Canet Juric, Richard's, Urquijo \& Burin, 2009). Además, respecto del monitoreo de la propia comprensión, los malos lectores tenían más fallas en una tarea de detección de errores, lo que señalaba que tenían menor capacidad para controlar metacognitivamente el proceso de comprensión detectando contradicciones y errores en la lectura (Canet Juric et al., 2009).

Otro aspecto a señalar es que, si bien cada una de estas habilidades resulta necesaria para lograr la comprensión, ninguna de ellas por sí sola puede garantizarla (Borzone de Manrique \& Signorini, 1994; Diuk \& Ferroni, 2009; Fumagalli, Wilson \& Jaichenco, 2010; Herrera \& Defior Citoler, 2005; Maldez, 2010; Oakhill, et al., 2003; Perfetti \& Hogaboam, 1975). Sobre las relaciones entre las mencionadas habilidades lectoras, se considera que decodificación y conocimiento léxico se hallan asociados y se influyen mutuamente (Mc Candliss et al., 2003; Perfetti, 2010a; Perfetti \& Hogaboam, 1975). En este sentido, los efectos de la decodificación en la comprensión 
global del texto se encuentran mediados por el conocimiento acerca del significado de las palabras y los efectos de la comprensión en la decodificación dependen de que se alcancen suficientes significados de palabras.

La decodificación y el acceso léxico de forma automática y fluída posibilitarían que los recursos de procesamiento estén disponibles para procesos de alto nivel en comprensión (Cain \& Oakhill, 2003; McCandliss et al., 2003; Nation, 2005; Perfetti \& Hogaboam, 1975; Perfetti \& Roth, 1980). Perfetti y Hogaboam (1975) encontraron que los lectores menos hábiles leían con mayor lentitud palabras y no palabras que el resto de sus compañeros de clase. Abordaron la decodificación de palabras y su producción, entendiendo por decodificación la transformación del código escrito en código oral y por producción, la vocalización de la unidad decodificada. En una muestra de niños de escolaridad primaria de tercero y quinto grado, identificaron a buenos y malos lectores. Presentaban a los niños tres clases de palabras y les solicitaban que las leyeran en voz alta: palabras comunes o frecuentes, palabras no tan frecuentes y pseudopalabras. En base al análisis de la variancia del tiempo que tardaban los niños en leer las palabras comunes, distinguieron a los buenos y malos lectores en cada grado. Los lectores hábiles presentaron tiempos de lectura significativamente menores que los malos lectores. Los lectores hábiles resultaron más rápidos en la decodificación oral de las palabras de baja frecuencia y de las pseudo-palabras, que los malos lectores. Esto se debería a que los lectores menos hábiles tendrían menos desarrolladas y automatizadas las habilidades de decodificación, en relación con los más hábiles. Además, los buenos comprendedores se habrían beneficiado con un vocabulario más amplio y variado, que fortalecería sus habilidades de decodificación y comprensión.

Teniendo en cuenta lo antes desarrollado, se realizó un estudio en una muestra de niños argentinos en la edad en que se suponen automatizados los procesos lectores de menor nivel (9-10 años), para investigar el estado y las relaciones entre las habilidades implicadas en la comprensión lectora (decodificación, conocimiento léxico e inferencias textuales) y estudiar la contribución de las habilidades de decodificación y conocimiento léxico a la realización de inferencias textuales.

Se enunciaron las siguientes hipótesis generales: (1) las habilidades de comprensión lectora se hallan relacionadas entre sí y (2) las habilidades de decodificación y conocimiento léxico son predictores significativos para la realización de inferencias textuales.

\section{MÉtodo}

\section{PARTICIPANTES}

La muestra estuvo compuesta por 94 alumnos de cuarto grado, de 8 a 10 años de edad: 49 eran alumnos de una escuela primaria suburbana de gestión estatal $(M=8.94)$ y 45 de una escuela primaria urbana $(M=8.93)$, ambas de la ciudad de San Miguel de Tucumán. A la escuela urbana asistían 24 niños y 21 niñas, y 38 de ellos (84.4\%) tenían 9 años de edad. A la escuela periurbana asistían 30 niños y 19 niñas, 36 de ellos (73.5\%) tenían 9 años de edad. La selección de los participantes se realizó teniendo en cuenta que las instituciones educativas representaran las zonas urbana y suburbana de la capital tucumana. Ambas escuelas se seleccionaron teniendo en cuenta las condiciones de vida de los habitantes de ambas zonas (DINIECE, 2007).

Se excluyeron de la investigación aquellos niños que tuvieran 3 años o más de sobreedad y que se encontraran en un proceso de integración escolar.

\section{INSTRUMENTOS}

Para obtener información sobre las habilidades de decodificación se administraron las pruebas de Identificación de Letras y de Procesos Léxicos de la Batería de Evaluación de los Procesos Lectores - PROLEC (versión original de Cuetos, Rodríguez, Ruano \& Arribas, 2010 y adaptación de Andrés, Canet Juric \& Introzzi, 2009). La prueba de Identi- 
ficación de Letras se compone de dos tareas: Nombre o Sonido de Letras, cuyo objetivo es comprobar si el niño conoce todas las letras y su pronunciación e Igual - Diferente, que busca conocer si el niño puede segmentar e identificar las letras que componen una palabra, a partir de la presentación de pares de palabras y pseudo-palabras iguales o diferentes en las que se cambia una de las letras. Una de las tareas de la Prueba de Procesos Léxicos es la Lectura de Pseudo-palabras o de Palabras Inexistentes, que mide la capacidad del lector para pronunciar palabras inexistentes, para lo cual tiene que emplear la vía de decodificación grafema - fonema.

Para evaluar las habilidades léxico - semánticas se aplicaron dos pruebas: (a) la adaptación de Contini, Figueroa, Cohen Imach y Coronel (2000) del Subtest de Vocabulario de la Escala de Inteligencia de Wechsler (WISC-III), que evalúa el caudal de palabras aprendido, para ello el niño debe definir palabras oralmente, y (b) la Tarea de Lectura de Palabras de la Prueba de Procesos Léxicos de la Batería PROLEC, cuyo objetivo es evaluar el reconocimiento y lectura de palabras ya que es uno de los procesos clave de la lectura y permite distinguir entre buenos y malos lectores. El niño debe leer palabras de alta y baja frecuencia de uso, cuya longitud varía entre 5 y 8 letras ( 2 a 3 sílabas). Las primeras sílabas constan de estructuras que combinan vocales $(\mathrm{V})$ y consonantes $(\mathrm{C})$, determinando diferentes grados de complejidad.

Para evaluar las habilidades inferenciales se administró la adaptación de Canet Juric y Burin (2006) de la Tarea de Inferencias de Cain \& Oakhill. La prueba consta de una historia de entrenamiento y cuatro historias de prueba, cada una con 13 ó 14 oraciones, seguidas de seis preguntas. Dos de ellas apuntan a la realización de inferencias puente y dos a la realización de inferencias elaborativas. Las dos restantes apuntan a la detección de información literal.

Con respecto a la confiabilidad de la adaptación del PROLEC (Andrés et al., 2009), la versión completa del test aplicada en una prueba piloto a 60 sujetos de escuelas muni- cipales de Mar del Plata y de 6 a 9 años de edad, arrojó un coeficiente de confiabilidad elevado $(\alpha=.72)$. Con respecto a la validez y a la estructura interna, el análisis factorial exploratorio de componentes principales permitió extraer cuatro factores que acumulados constituían el 79.18\% de la variancia total explicada ${ }^{1}$.

En relación con el Subtest de Vocabulario del WISC-III, el instrumento en general fue analizado mediante el método de división por mitades. Se correlacionaron los puntajes de los dos medios y el coeficiente resultante fue corregido mediante la fórmula SpearmanBrown, arrojando un coeficiente de confiabilidad alto $(r=.87)$ para el mencionado subtest. Además, y haciendo referencia a la validez, este instrumento se encuentra relacionado con el factor denominado Comprensión Verbal del WISC-III y ha demostrado ser sensible para grupos de niños dotados y con dificultades de aprendizaje (Wechsler, 1991). Por otra parte, cabe señalar que para población tucumana se cuenta con el baremo local del instrumento general, elaborado por Contini y colaboradores (2000).

1 Sólo se analizaron las escalas que tenían una carga superior a .62. El Factor 1 de mayor carga, que explica el $55.90 \%$ de la variancia, obtuvo saturaciones altas en las variables: decisión léxica (palabrasno palabras), lectura de palabras y de pseudopalabras, estructuras gramaticales (activas - pasivas complemento focalizado) y comprensión de oraciones. El Factor 2 está conformado por las variables relacionadas con la comprensión de textos (narrativos, expositivos, preguntas literales y preguntas inferenciales). El Factor 3 posee saturaciones altas en la variable signos de puntuación (coma, punto, interrogación y exclamación) y el Factor 4 arrojó el menor porcentaje de variabilidad explicada incluyendo las cargas de las variables Nombres de Letras e Igual - Diferente (subescala igual y subescala diferente). El valor alpha de .62 de Nombre - Sonido de las letras en niños de 9 años puede deberse a que esta escala es la más sencilla de todas y requiere sólo identificación de letras, de manera que en niños de mayor edad perdería poder discriminativo. 
Dado que la calificación de la adaptación de la Tarea de Inferencias de Cain y Oakhil (2006) implica cierto grado de subjetividad, 34 protocolos fueron puntuados por cuatro jueces, y el acuerdo inter-jueces para cada ítem fue evaluado con el estadístico Kappa de Cohen (Cohen, 1960). El valor medio de Kappa fue igual a .74, lo cual indica un buen nivel de acuerdo. Además, el instrumento final presenta un adecuado nivel de consistencia interna $(\alpha=.728)$.

\section{Procedimiento}

Se solicitó autorización a los directivos de las instituciones educativas para realizar este estudio, explicitando los propósitos del mismo y el tipo de materiales a utilizar. También se pidió el consentimiento informado a los padres para evaluar a los niños.

Se realizó una prueba piloto a fin de estimar si el procedimiento de administración era el adecuado y cómo los niños respondían a las pruebas escogidas. La administración definitiva fue individual y se organizó de la siguiente manera: en primer lugar se administraron las tareas del PROLEC y el Subtest de Vocabulario. Luego los niños descansaron durante 20 minutos, en los cuales realizaron actividades lúdicas. Posteriomente se administraron las tareas de inferencias. Finalmente, se efectuó la devolución de los resultados a las instituciones educativas participantes, a través de talleres en los espacios institucionales de coordinación y planificación escolar.

\section{Resultados}

La Decodificación se estimó a partir de la Identificación de Letras y de la Lectura de Pseudo-palabras; el Conocimiento Léxico, a través de la Lectura de palabras y de Vocabulario y la comprensión de texto, por la capacidad para realizar inferencias.

En la Tabla 1 se presentan los estadísticos descriptivos de la muestra para las habilidades y subhabilidades de la comprensión lec- tora abordadas en este estudio. Como se observa en esta tabla, para Decodificación, la media más alta de errores se aprecia en Lectura de pseudo-palabras $(M=5.86)$ y la media más alta de aciertos $(M=34.16)$ en esta misma prueba. Para Conocimiento léxico, la media más alta de aciertos se observa en la Lectura de palabras $(M=35.77)$. En cuanto a los Procesos de integración textual, en inferencias la media de errores es igual a 14.62. Como se observa, las pruebas utilizadas miden errores y aciertos, por lo que se construyeron índices de decodificación y de conocimiento léxico.

Como se indicó acerca de las habilidades lectoras, se construyó un índice de decodificación en base al promedio del puntaje $z$ en las medidas de errores en Reconocimiento de letras, en Igual - Diferente y en Lectura de no palabras. Este puntaje se denomina $z$ decodificación y es el puntaje estándar de errores promedio en estas tres medidas (puntaje inverso). También se construyó un índice de conocimiento léxico a partir del promedio de los puntajes estandarizados en Lectura de palabras y en Vocabulario. Este puntaje se denomina $z$ conocimiento léxico.

Para estudiar las relaciones entre las habilidades lectoras implicadas en la comprensión lectora, se analizaron las correlaciones entre las diferentes habilidades lectoras evaluadas (Decodificación, Conocimiento léxico, Inferencias puente e Inferencias elaborativas) que se presentan en la Tabla 2. Se observan correlaciones medias y negativas de la Decodificación dado que es un puntaje inverso con las inferencias en general $(r=-.59)$, las Inferencias puente $(r=-.58)$, las Inferencias elaborativas $(r=-.54)$ y considerables con el Conocimiento léxico $(r=.82)$. El Conocimiento léxico presentó correlaciones significativas y positivas de tamaño considerable con las inferencias en general $(r=.72)$ y de tamaño mediano con las Inferencias puente $(r=.69)$ y las Inferencias elaborativas $(r=.54)$. Las inferencias, en general, correlacionan positiva y fuertemente entre sí $(r=.83)$, positiva y considerablemente con el Vocabulario $(r=.67) \mathrm{y}$ negativa y considerablemente con la Decodificación $(r=-.77)$. 
Dadas estas correlaciones, la relación de precedencia temporal de las variables Decodificación y Conocimiento léxico sobre la actividad inferencial y la literatura informada en la Introducción, se examinó la contribución de las primeras sobre las inferencias a partir de regresiones lineales. Sobre el total de inferencias se ingresaron los índices de Decodificación y Conocimiento léxico como predictores, y el total de inferencias y de las Inferencias puente y elaborativas como criterios. Se ingresaron ambas como predictores (método Enter).

Sobre el total de inferencias, el conjunto de variables explicó un 51\% de las Inferencias $[F(2)=49.36, p<.01]$. Entre los predictores, el único que realizó una contribución significativa fue el Conocimiento léxico $[\beta$ estandarizado $=.72, t(58)=5.6, p<.01]$.

Se examinaron por separado las inferencias elaborativas y puente. Se repite el patrón de resultados: en ambos casos, se explicó una proporción significativa de la variancia y el predictor que realizaba una contribución significativa era el Conocimiento léxico. El análisis no se ve afectado por la colinealidad, que resulta baja (.321). En el caso de las Inferencias puente, el conjunto de las variables explicó el $48 \%$ de éstas $[F(2)=43.24, p<.01]$, el único predictor que realizó una contribución significativa fue el Conocimiento léxico [ $\beta$ estandarizado $=.66, t(-25)=-5.00, p<.01] \mathrm{y} \mathrm{el}$ análisis no se vio afectado por la colinealidad, que resultaba baja (.321).

En cuanto a las Inferencias elaborativas, el conjunto de las variables explicó el $45 \%$ de éstas $[F(2)=39.95, p<.01]$, el único predictor que hizo una contribución significativa fue también el Conocimiento léxico [ $\beta$ estandarizado $=-.72, t(-24)=-5.36, p<.01]$ y el análisis no se vio afectado por la colinealidad, que resultaba baja (.321).

\section{DISCUSIÓN}

El problema abordado en el estudio realizado se centró en las dificultades en comprensión lectora de niños de 9 a 10 años de edad de la ciudad de San Miguel de Tucumán, considerando las variables psicolingüísticas implicadas. La elección de la temática respondió a la necesidad de contar con estudios locales que indagaran las habilidades implicadas en la comprensión textual, a una edad en la que se espera que éstas se hayan desarrollado. Así, uno de los propósitos como ya se indicó, fue estudiar las relaciones entre las habilidades implicadas en la comprensión lectora (decodificación, conocimiento léxico, comprensión verbal e inferencias textuales) (hipótesis 1).

Se observaron correlaciones de tamaño moderado a alto y significativas entre las habilidades que componen la comprensión lectora. En línea con la propuesta de Perfetti (2010a), las habilidades decodificadoras correlacionaron significativamente con el vocabulario y con la comprensión verbal; el vocabulario correlacionó significativamente con las inferencias y con la comprensión verbal; la comprensión verbal correlacionó con el vocabulario y las inferencias y estas correlacionaron significativamente entre sí (Inferencias puente y elaborativas) con el vocabulario. Además, Conocimiento léxico e Inferencias puente y elaborativas evidenciaron correlaciones significativas entre sí, lo cual se sitúa también en la misma línea de lo señalado por Perfetti (2010a), ya que para este autor Decodificación, Conocimiento léxico e Inferencias son factores correlacionados.

Con respecto a la contribución de las habilidades de Decodificación, Conocimiento léxico y Conocimiento previo lingüístico en la realización de inferencias textuales (hipótesis 2), en función de la regresión lineal, se observó que las habilidades lectoras básicas lograban explicar la realización de inferencias y que el Conocimiento léxico se presentaba como la habilidad que permitía predecir la posibilidad de que los niños realicen inferencias. En el caso de los tipos de inferencias, el Conocimiento léxico se presentó también como un factor que permitía explicar la activación de los procesos inferenciales y predecir la realización de Inferencias puente y elaborativas en medidas similares. Esto se sitúa en la línea de la hipótesis según la cual se observaría una contribución significativa de las 
habilidades de Conocimiento léxico en la realización de Inferencias textuales.

La regresión de las variables de lectura sobre las inferencias mostró que el Conocimiento léxico parece incidir directamente en la posibilidad de realizar inferencias. Esto coincide con lo propuesto por Perfetti (2010a), quien supuso que la Decodificación y el vocabulario son habilidades interdependientes y que la relación entre Decodificación y habilidades inferenciales se encuentra mediada por el vocabulario.

Otro análisis concierne a los resultados descriptivos de la muestra, en comparación con otros estudios nacionales. Los resultados de la muestra general, en cuanto a la Decodificación evidenciaron por una parte, que el rendimiento fue ligeramente superior al descripto por Andrés y colaboradores (2009) y Urquijo y colaboradores (2006), con niños de 6 a 8 años de edad de escuelas de gestión pública. Además, el rendimiento en Lectura de palabras y de pseudo-palabras fue significativamente inferior al señalado en los estudios antes referidos. Acerca del vocabulario, el rendimiento fue similar al hallado en otros estudios (Contini et al., 2000) realizados con niños de edades similares a los de la presente investigación (8 y 9 años de edad) y de escuelas estatales urbanas y suburbanas. En cuanto a las inferencias, el desempeño fue análogo al señalado por Canet Juric y colaboradores (2007) en un estudio realizado con niños de 8 y 9 años de edad de una escuela de gestión estatal urbana.

En general, de esto se desprende que el rendimiento de la muestra general en las habilidades lectoras evaluadas según las pruebas utilizadas, se asemeja al de poblaciones infantiles de las mismas edades estudiadas en nuestra provincia y en otras provincias del país.

En este sentido, el estudio realizado aporta evidencias empíricas a favor de las hipótesis planteadas, a saber: (1) las habilidades de la comprensión lectora se hallan relacionadas entre sí y (2) las habilidades de Decodificación, Conocimiento léxico y conocimiento general inciden en la realización de inferencias textuales.

En relación con lo expuesto, cabe señalar que tener en cuenta cómo las habilidades lectoras se relacionan entre sí y se ven afectadas, permitiría desarrollar programas de intervención a fin de mejorar el rendimiento de aquellos niños que presenten dificultades lectoras. Sin embargo, es importante señalar que las conclusiones y los alcances de esta investigación deben interpretarse teniendo en cuenta que sólo se estudiaron niños de 9 años.

Acorde a lo propuesto por Cain y Oakhill (2003), por Nation (2005) y por Perfetti y colaboradores (2001), investigaciones posteriores podrían evaluar los procesos metacognitivos o de orden superior o de monitoreo, que los sujetos pueden implementar espontánea y voluntariamente mientras escuchan o leen, a fin de estimar si comprenden correctamente el discurso. Asimismo, y siguiendo a Cain y Oakhill (1999) podría evaluarse la sensibilidad a la estructura de la historia. En concordancia con la propuesta de diversos estudios (Marshall \& Nation, 2003; Nation, Adams, Bowyer-Crane \& Snowling, 1999; Seigneuric et al., 2000; Yuill et al., 1989), investigaciones futuras podrían indagar la relación entre la comprensión textual y la memoria de trabajo.

Además se podrían evaluar las habilidades lectoras de forma longitudinal en todos los cursos de la escolaridad primaria a fin de contar con información que permita comparar las habilidades lectoras desarrolladas por los niños en su proceso de alfabetización básica. También sería posible indagar en estudios sucesivos las habilidades cognitivas generales de los niños que viven en condiciones de pobreza y su relación con la comprensión textual. 
TABLA 1

ESTADÍSTICOS DESCRIPTIVOS DE LA MUESTRA PARA LAS HABILIDADES DE COMPRENSIÓN LECTORA

\begin{tabular}{||l|l|c|cc|cc|}
\hline \multicolumn{1}{|c|}{ Habilidad } & \multicolumn{1}{|c|}{ Sub-habilidad } & Índice & Mín. & Máx. & $M$ & DE \\
& & & & & \\
& \multirow{2}{*}{ Reconocimiento de letras } & TE & 0 & 8 & 2.88 & 1.765 \\
& TA & 12 & 20 & 17.12 & 1.765 \\
& Igual - Diferente & TE & 0 & 9 & 3.63 & 2.084 \\
& & TA & 0 & 20 & 16.16 & 2.653 \\
& Lectura de pseudo-palabras & TE & 1 & 10 & 5.86 & 2.686 \\
& & TA & 30 & 39 & 34.16 & 2.693 \\
\hline \multirow{2}{*}{ Conocimiento léxico } & Vocabulario & TA & 4 & 15 & 9.97 & 3.198 \\
& Lectura de palabras & TE & 0 & 10 & 4.23 & 2.807 \\
& & TA & 30 & 40 & 35.77 & 2.807 \\
\hline Procesos de & Inferencias (errores) & TE & 4 & 24 & 14.62 & 5.948 \\
\hline integración textual & & & & & & \\
\hline
\end{tabular}

TABLA 2

CORRELACIONES ENTRE LAS MEDIDAS DE LECTURA

\begin{tabular}{||l|c|c|c|c|c|}
\hline \multicolumn{1}{|c|}{ Variables } & Decodificación & $\begin{array}{c}\text { Conocimiento } \\
\text { léxico }\end{array}$ & $\begin{array}{c}\text { Inferencias en } \\
\text { general }\end{array}$ & $\begin{array}{c}\text { Inferencias } \\
\text { puente }\end{array}$ & $\begin{array}{c}\text { Inferencias } \\
\text { elaborativas }\end{array}$ \\
\hline Decodificación & $.824^{* *}$ & $-.595^{* *}$ & $-.721^{* *}$ & & \\
Conocimiento léxico & $-.589^{* *}$ & $-.698^{* *}$ & $.967^{* *}$ & & \\
Inferencias en general & $-.547^{* *}$ & $-.683^{* *}$ & $.947^{* *}$ & $.834^{* *}$ & \\
\hline
\end{tabular}

Inferencias: puntaje de error (inverso)

${ }^{* *} p<.01$ 


\section{REFERENCIAS BIBLIOGRÁFICAS}

Abusamra, V., Ferreres, A. \& Raiter, A. (2010). Las habilidades de comprensión. Un enfoque cognitivo [Comprehension skills. A congnitive frame]. En V. Abusamra, A. Ferreres, A. Raiter, R. De Beni \& C. Cornoldi (Eds.), Test Leer para Comprender. TLC. Evaluación de la comprensión de textos (pp. 25-45). Buenos Aires: Paidós.

Andrés, M.L., Canet Juric, L. \& Introzzi, I. (2009). Adaptación de una prueba para evaluar lectura: El problema de las inferencias [Adaptation of a reading comprehension test: The trouble of inferences]. Revista Científica Electrónica de Psicología, 5, 143-158.

Belinchón, M., Igoa, J.M. \& Riviére, A. (1992). La comprensión del discurso [Discourse comprehension]. En M. Belinchón, J. Igoa \& A. Riviére (Eds.), Psicología del lenguaje. Investigación y teoría. Madrid: Trotta.

Booth, J.R., MacWhinney, B. \& Perfetti, C.A. (1999). Quick, automatic, and general activation of orthographic and phonological representations in young readers. Developmental Psychology, 35(1), 3-19. http://dx.doi.org/10. 1037\%2F\%2F0012-1649.35.1.3

Borzone, A.M. (2005). La resolución de anáforas en niños: Incidencia de la explicitud y de la distancia [Anaphor resolution in children: Incidence of explicitness and distance]. Interdisciplinaria, 22(2), 155-182. Recuperado el 3 de febrero de 2012 de http://redalyc.uaemex.mx/ src/inicio/ArtPdfRed.jsp?iCve=18022203

Borzone, A.M. \& Silva, M.L. (2007). La resolución de anáforas en niños: Incidencia de la causalidad implícita de los verbos [Anaphor resolution in children: Incidence of the implicit causality of verbs]. Límite, 2, 81-100.

Borzone de Manrique, A.M. \& Signorini, A. (1994). Phonological awarness, spelling and reading abilities in Spanish-speaking children. British Journal of Educational Psychology, 64, 429-439.

Cain, K. \& Oakhill, J. (1999). Inference making ability and its relation to comprehension failure in young children. Reading and Writing: An Interdisciplinary Journal, 1(5), 489-503.

Cain, K. \& Oakhill, J. (2003). Reading comprehension difficulties. En T. Nunes \& P. Bryant (Eds.), Handbook of children's literacy (pp. 313-338). Netherlands: Dordretch.

Cain, K., Oakhill, J.V., Barnes, M.A. \& Bryant, P.E. (2001). Comprehension skill, inference making ability, and their relation to knowledge. Memory and Cognition, 29, 850-859.

Cain, K., Oakhill, J. \& Bryant, P. (2000). Investigating the causes of reading comprehension failure: The comprehension-age match. Reading and Writing: An Interdisciplinary Journal, 12 (1), 31-40. Recuperado el 10 de diciembre de 2010 de http://www.psych.lancs.ac.uk/people/ KateCain.html.

Cain, K., Oakhill, J. \& Bryant, P. (2003). Children's reading comprehension ability: Current prediction by working memory, verbal ability, and component. Journal of Educational Psychology, 96(1), 31-42. Recuperado el 12 de noviembre de 2010 de http://www.psych.lan cs.ac.uk/people/uploads/KateCain20050929T1 05101.pdf

Canet Juric, L. (2009). Análisis de una tarea de inferencias y una tarea de monitoreo: Reporte de resultados de prueba [Analysis of an inferences task and a monitoring task: Report of test results]. Anuario de Proyectos e Informes de Investigación de Becarios, 6, 195-203.

Canet Juric, L., Andres, M.L., Urquijo, S. \& Ane, A.M. (2007). Métodos de enseñanza de la lectoescritura y desempeño académico [Methods of teaching literacy and academic performance]. En Universidad Nacional de Catamarca (Ed.), Lectura y escritura. Caminos para la 
construcción del mundo (pp. 684-693). Catamarca: Editorial Científica Universitaria.

Canet Juric, L. \& Burin, D. (2006). Adaptación de las Tareas de Inferencias de Cain \& Oakhill [Cain \& Oakhill' Inferences Task Adaptation]. Manuscrito no publicado.

Canet Juric, L., Richard's, M., Urquijo, S. \& Burin, D. (2009). Predictores cognitivos de niveles de comprensión lectora mediante análisis discriminante [Cognitive predictors of reading comprehension levels using discriminant analysis]. International Journal of Psychological Research, 2, 99-111.

Cohen, J. (1960). A coefficient of agreement for nominal scales. Educacional and Psychological Measurement, 20(4), 347-372.

Contini, E.N., Figueroa, I., Cohen Imach, S. \& Coronel de Pace, P. (2000). Habilidades cognitivas en niños y adolescentes. Evaluación psicológica desde una perspectiva socio-cultural [Cognitive skills in children and adolescents. Psychological assessment in a socio-cultural perspective]. San Miguel de Tucumán: Magna.

Cuetos, F., Rodríguez, B., Ruano, E. \& Arribas, D. (2010). Batería de Evaluación de los Procesos Lectores, Revisada [Revised Assessment Battery of Reading Processes, PROLEC-R]. Madrid: TEA.

DINIECE - Dirección Nacional de Información y Evaluación de la Calidad Educativa (2007). Operativo Nacional de Evaluación 2007 [Evaluation National Assessment 2007]. Recuperado el 9 de marzo de 2010 de http://dinie ce.me.go v.ar/images/stories/diniece/evaluacion_educat iva/nacionales/resultados/Estudio\%20Nacional $\% 20 \mathrm{de} \% 20$ Evaluacion $\% 20 \mathrm{y} \% 20$ Consideracio nes\%20conceptuales\%20ONE\%20200 7.pdf

Diuk, B. \& Ferroni, M. (2009). Dificultades lectoras en niños que crecen en contextos de pobreza: Perfiles cognitivos de niños con y sin dificultades [Reading difficulties in children growing up in poverty contexts: Cognitive profiles of child- ren with and without difficulties]. Cuadernos de Psicopedagogía, 7(13). Recuperado el 6 de agosto de 2010 de http://pepsic.bvsalud.org/sc iel o.php?pid=S1676-10492009000100005\&sc ript $=$ sci_arttext

Diuk, B., Signorini, A. \& Borzone, A.M. (2003). Las estrategias de lectura de palabras en niños de $1^{\circ}$ a $3^{\circ}$ año de Educación General Básica: Un estudio comparativo entre niños procedentes de diferentes sectores sociales [Word reading strategies in1st to 3rd year children from Basic General Education: A comparative study between children from different social sectors]. Psykhe, 12, 29-48.

Ferreres, A., Abusamra, V., Casajús, A. \& China, N. (2011). Adaptación y estudio preliminar de un test breve para evaluar eficacia lectora (TECLE) [Adaptation and preliminary study of a brief test to assess reading efficacy (TEC LE)]. Revista Neuropsicológica Latinoamericana, 3, 1-7. Recuperado el 28 de febrero de 2012 de http://neuropsicolatina.org/index.php/ Neuropsicologia_Latinoamericana/article/vie $\mathrm{w} / 40 / 42$

Ferreres, A., Abusamra, V. \& Squillace, M. (2010). Comprensión de textos y oportunidades educativas [Text comprehension and educational opportunities]. En Organización de Estados Iberoamericanos (Ed.), Actas del Congreso Iberoamericano de Educación. Metas 2021 (pp. 1-11). Buenos Aires: OEI. Recuperado el 29 de febrero de 2012 de http://www.ade epra.org.ar/cong resos/Congreso\%20IBEROAMERICANO/ COMPETENCIASBASICAS/RLE2515bCarto ceti.pdf

Fumagalli, J., Wilson, M. \& Jaichenco, V. (2010). Sensibilidad a la información fonológica en niños lectores y prelectores hispanoparlantes [Sensitivity to phonological information in reading and pre-reading Spanish-speaking children]. Revista Neuropsicológica Latinoamericana, 2, 68-77. 
Gernsbacher, M.A.,Varner, K.R. \& Faust, M.R. (1990). Investigating differences in general comprehension skill. Journal of Experimental Psychology, 16(3), 430-445. Recuperado el 15 de septiembre de 2010 de http://128.104.13 0.43/lang/pdf/Gernsbacher_GeneralCompSkill JEPLMC_1990.pdf.

http://dx.doi.org/10.1037\%2F\%2F0278-7393. 16.3.430

Gutierrez, F., Elosúa, M.R., García Madruga, J.A. Gárate, M. \& Luque (1999). Memoria operativa y comprensión lectora [Working memory and reading comprehension]. En J. García Madruga, M.R. Elosúa, F. Gutierrez, J. Luque \& M. Gárate (Eds.), Comprensión lectora y memoria operativa. Aspectos evolutivos e instruccionales (pp. 15-31). Barcelona: Paidós.

Herrera, L. \& Defior Citoler, S. (2005). Una aproximación al procesamiento fonológico de los niños prelectores: Conciencia fonológica, memoria verbal a corto plazo y denominación [An approach to phonological processing in preliterate children: Phonological awareness, verbal short-term memory and naming]. Psykhe, 14, 81-95. Recuperado el 23 de febrero de 2011 de http://www.scielo.cl/scielo.php?pid=S0718-2 2282005000200007\&script= sci_ar ttext\# back Hulme, C., Snowling, M., Caravolas, M. \& Carroll, J. (2004). Phonological skills are (probably) one cause of success in learning to read: A comment on Castles and Coltheart. Scientific Studies on Reading, 9(4), 351-365. Recuperado el 10 de septiembre de 2010 de http://web.ebscohost.co $\mathrm{m} /$ ehost $/$ pdfviewer/pdfviewer?hi $\mathrm{d}=113 \&$ sid $=$ 263d317d-b01a-4bc9-8bc3-e666d45a7ccc\% 40sessionmgr $113 \&$ vid $=10$

Kintsch, W. (1998). The role of knowledge in discourse comprehension: A construction-integration model. Psychological Review, 85, 363394.

León, J.A. (2003). Una introducción a los procesos de inferencia en la comprensión del discurso escrito [An introduction to inferential processes in the comprehension of written discourse]. En J.A. León (Ed.), Conocimiento y discurso (pp. 23-43). Madrid: Pirámide.

León, J.A. (2004). ¿Por qué las personas no comprenden lo que leen? [Why do not people understand what they read?]. Psicología Evolutiva, 10(2), 101-116. Recuperado el 12 de noviembre de 2010 de http://web.ebscohost.com/ ehost $/ \mathrm{p}$ dfviewer/pdfvie wer?hid=113\& sid = 263d317d-b01a-4bc9-8bc3-e666d45a7ccc\% 40session mgr113\&vid=10.

Liberman, I.Y., Shankweiler, D. \& Liberman A.M. (1989). The alphabetic principle and learning to read. En I.Y. Liberman, D. Shankweiler \& A.M. Liberman (Eds.), Phonology and reading disability. Solving the reading puzzle (pp. 1-34). Michigan: National Institute of Child Health and Human Development.

Maldez, M. (2010). Conciencia fonológica y aprendizaje [Learning and phonological awarness]. En M. Viramonte de Ávalos (Dir.), Salud y aprendizajes lingüísticos. Complejidades en la enseñanza de la lengua. Estudios y propuestas de acciones superadoras. Tomo II (pp. 343373). Córdoba: Universidad Blas Pascal, Universidad Nacional de Río Cuarto, Ministerio de Ciencia y Tecnología, Agencia Nacional de Promoción Científica y Tecnológica.

Marder, S.E. (2011). Resultados de un programa de alfabetización temprana. Desempeño en lectura en niños de sectores en desventaja socioeconómica [Results of an early literacy intervention program. Reading skills in children with socio-economic disadvantages]. Interdisciplinaria, 28(1), 159-176.

McCandliss, B., Beck, I.L., Sandak, R. \& Perfetti, C. (2003). Focusing attention on decoding for children with poor reading skills: Design and preliminary tests of the word building intervention. Scientific Studies of Reading, 7(1), 75104. Recuperado el 10 de noviembre de 2010 
de http://web.ebscohost.com/ehost/pdfviewer/ pdfviewer?hid $=113 \&$ sid $=263 \mathrm{~d} 317 \mathrm{~d}-\mathrm{b} 01 \mathrm{a}-4 \mathrm{~b}$ c9-8bc3-e666d45a7ccc\%40sessionmgr113\& vid $=10$ http://dx.doi.org/10.1207\%2FS1532799XSSR 0701_05

Marshall, C.M. \& Nation, K. (2003). Individual differences in semantic and structural errors in children's memory for sentences. Educational and Child Psychology, 20(3), 7-18. Recuperado el 28 de noviembre de 2010 de http://www. bps.org.uk/downloadfile.cfm?file_uuid=3B471 D39-1143-DFD0-7E6C45DE7B35E2FB\&ex $\mathrm{t}=$ pdf\#page $=9$

Molinari, C. (2008). Introducción a los modelos cognitivos de la comprensión del lenguaje [Introduction to reading comprehension cognitive models]. Buenos Aires: Eudeba.

Nation, K. (2005). Children's reading comprehension difficulties. En M.J. Snowling \& C. Hulme (Eds.), The science of reading: A handbook (pp. 248-265). Oxford: Blackwell.

Nation, K., Adams, J., Bowyer-Crane, C. \& Snowling, M. (1999). Working memory deficits in poor comprehenders reflect underlying language impairments. Journal of Experimental Child Psychology, 73(2), 139-158.

Nation, K. \& Snowling, M. (1998). Semantic processing and the development of word recognition skill: Evidence from children with reading comprehension difficulties. Journal of Memory and Language, 39, 85-101. Recuperado el 7 de noviembre de 2010 de http://www.haskins. yale.edu/papers/Nation_and_Snowling_1998.p df.

http://dx.doi.org/10.1006\%2Fjm la.1998.2564

Nation, K. \& Snowling, M. (2004). Beyond phonological skills: Broarder language skills contribute to the development of reading. Journal in Research of Reading, 27(4), 342-356. Recuperado el 8 de noviembre de 2010 de http://web. ebscohost.com/ehost/pdfviewer/pdfviewer?hid
$=113 \&$ sid $=263 \mathrm{~d} 317 \mathrm{~d}-\mathrm{b} 01 \mathrm{a}-4 \mathrm{bc} 9-8 \mathrm{bc} 3-\mathrm{e} 666 \mathrm{~d} 4$ $5 \mathrm{a} 7 \mathrm{ccc} \% 40$ sessionmgr13\&vid $=10$. http://dx.doi.org/10.1111\%2Fj.1467-9817.200 4.00238.x

Oakhill, J. (1982). Constructive processes in skilled and less skilled comprehenders'memory for sentences. British Journal of Educational Psychology, 73, 13-20. http://dx.doi.org/10.1111\% 2Fj.2044-8295.198 2.tb01785.x

Oakhill, J. (1983). Instantiation in skilled and less skilled. Quaterly Journal of Experimental Psychology, 35, 441-450. http://dx.doi.org/10.10 80\%2F14640748308402481

Oakhill, J., Cain, K. \& Bryant, P. (2003). The dissociation of word reading and text comprehension: Evidence from component skills. Language and Cognitive Processes, 18(4), 443468. Recuperado el 30 de noviembre de 2010 de http://web.ebscohost.com/ehost/pdfviewer/ pdfviewer?hid=12\&sid=0a405bc8-789e-498086b6-a9a18bb8bafb\%40sessio nm gr115\&vid $=5$.

http://dx.doi.org/10.1080\%2F0169096034400 0008

Parodi, G., Peronard, M. \& Ibañez, R. (2010). ¿Qué es saber leer? [What is knowing to read?]. En G. Parodi, M. Peronard \& R. Ibañez (Eds.), Saber leer (pp. 19-55). Madrid: Santillana.

Perfetti, C. (2010a). Decoding, vocabulary and comprehension. The golden triangle of reading skill. En M.G. McKeown \& L. Kucan (Eds.), Bringing reading research to life (pp.291-303). New York: Guilford. Recuperado el 3 de febrero de $2011 \mathrm{de}$ http://www.pitt.edu/ perfetti/P DF/Decoding\%20Beck\%20festschrift\%20cha pt.pdf

Perfetti, C.A. (2010b). Reading. En P.C. Hogan (Ed.), Cambridge encyclopedia of the language sciences (pp. 699-702). Storrs, C.T.: University of Connecticut. Recuperado el 15 de octubre de 2011 de http://www.pitt.edu/ perfetti/PDF/ Reading\%20Cambridge\%20699-702.pdf 
Perfetti, C. \& Hogaboam, T. (1975). Relationship between single word decoding and reading comprehension skill. Journal of Educational Psychology, 67(4), 461-469. http://dx.doi.org/ $10.1037 \% 2 \mathrm{Fh} 0077013$

Perfetti, C., Landi, N. \& Oakhill, J. (2005). The acquisition of reading comprehension skill. En M.J. Snowling \& C. Hulme (Eds.), The science of reading: A handbook (pp. 227-247). Oxford: Blackwell.

Perfetti, C.A. \& Roth, S. (1980). Some of the interactive processes in reading and their role in reading skill. En M. Leslong \& C. Perfetti (Eds.), Processes in reading (pp. 1-52). Pittsburg: Erlbaum. Recuperado el 10 de noviembre de 2010 de http://eric.ed.gov/PDFS/ED19848 2.pdf

Perfetti, C.A., Van Dyke, J. \& Hart, L. (2001). The psycholinguistics of basic literacy. Annual Review of Applied Linguistics, 21, 127-149. Recuperado el 16 de noviembre de 2010 de http:// w ww.pitt.edu/ perfetti/PDF/Psycholinguistics $\% 2$ 0 of $\% 20$ basic $\% 201$ iteracy-\%20Van $\% 2$ 0Dyke, $\% 20$ Hart.pdf.

PISA - Programme for International Student Assessment (2009). Resumen ejecutivo, Argentina. Subsecretaria de Planeamiento [Executive Summary, Argentina. Undersecretary of Planning]. Buenos Aires: Dirección Nacional de Información y Evaluación de la Calidad Educativa. Ministerio de Educación de la Nación.

PISA (2012). Programa internacional de evaluación de estudiantes. Lengua. Subsecretaría de Planeamiento [International programme of students evaluation. Language. Undersecretary of Planning]. Buenos Aires: Dirección Nacional de Información y Evaluación de la Calidad Educativa. Ministerio de Educación de la Nación.

Plana, M.D. \& Fumagalli, J. (2013). Habilidades y conocimientos constitutivos de la alfabetización temprana: Semejanzas y diferencias según el entorno social y las oportunidades educativas
[Skills and knowledge constitutive of early literacy: Similarities and differences by social environment and educational opportunities]. Interdisciplinaria, 30(1), 5-24.

Seigneuric, A.S, Ehrlich, M.F., Oakhill, J.V. \& Yuill, N.M. (2000). Working memory resources and children's reading comprehension. Reading and Writing, 13, 81-103.

Serrano, M.S. \& Canigia M.L. (2009). Análisis de resultados del área lengua [Analysis of results of the language area]. En Ministerio de Educación de Tucumán (Ed.), Presentación de los resultados del Operativo Provincial de Evaluación de Calidad Educativa para EGB 2 (pp. 27-164). Tucumán: Ministerio de Educación de Tucumán.

Shankweiler, D., Lundquist, E., Katz, L., Stuebing, K.K., Fletcher, J.M., Bardy, S., Fowler, A., Dreyer, L.G., Marchione, K.E., Shaywitz, S.E. \& Shaywitz, B.A. (1999). Comprehension and decoding: Patterns of association in children with reading difficulties. Scientific Studies of Reading, 3(1), 69-94. Recuperado el 5 de noviembre de 2010 de http://www.has kins.yale. edu/Reprints/HL1103.pdf. http://dx.doi.org/10.1207\%2Fs1532799xssr030 14

Urquijo, S. (2009). Aprendizaje de la lectura. Diferencias entre escuelas de gestión pública y de gestión privada [Learning to read. Differences between public and private schools]. Evaluar, 9, 19-34. Recuperado el 7 de abril de 2011 de http://revistas.unc.edu.ar/index.php/revaluar/ar ticle/view/462

Urquijo, S., Canet Juric, L., Andrés, M.L. \& Ané, A. (2006). Los procesos lectores en primer ciclo de EGB (Informe técnico para la Municipalidad de Pueyrredón) [Reading processes in the EGB first cicle (Technical report for Pueyrredón Municipality)]. Mar del Plata, Buenos Aires: Universidad Nacional de Mar del Plata, Grupo de Psicología Cognitiva y Educacional. 
Wechsler, D. (1991). Test de Inteligencia para $\mathrm{Ni}$ ños. WISC-III. Manual [Intelligence Scale for children. WISC-III. Manual]. Buenos Aires: Paidós.

Yuill, N., Oakhill, J. \& Parkin, A. (1989). Working memory, comprehension ability and the reso- lution of text anomaly. British Journal of Psychology, 80(3), 351-361. Recuperado el 30 de noviembre de 2010 de http://web.ebscohost.co $\mathrm{m} /$ ehost $/$ pdfviewer/pdfviewer?hid $=12 \&$ sid $=0 \mathrm{a}$ 405bc8-789e-4980-86b6-a9a18bb8bafb\%4 0sessionmgr115\&vid=11.

Instituto de Investigaciones del Lenguaje y la Cultura (INVELEC) Facultad de Filosofía y Letras Universidad Nacional de Tucumán (UNT) Consejo Nacional de Investigaciones Cientificas y Técnicas (CONICET)

San Miguel de Tucumán Tucumán - República Argentina

Fecha de recepción: 3 de octubre de 2013 Fecha de aceptación: 2 de junio de 2014 